\title{
Deformation Simulation of Human Kidney Using Molecular Modelling Method
}

\author{
Alpaslan Duysak ${ }^{\mathrm{a}, \mathrm{b} *}$, Mustafa M. Atanak ${ }^{\mathrm{a}, \mathrm{b}}$, Ebru Duysak $\mathrm{k}^{\mathrm{a}, \mathrm{b}}$ \\ ${ }^{a}$ Computer Engineering, Dumlupinar University, Kutahya, Turkey

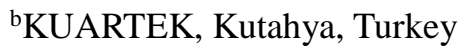 \\ *Corresponding Author: aduysak@dpu.edu.tr
}

\begin{abstract}
This paper focuses on modelling and simulation of the interaction between human kidney model and a surgical tool, Nephroscope. A surface model of a kidney with 1.986 vertices and 3.968 triangles is used in the deformation simulations. The simulation algorithm detects the collision between Nephroscope and kidney surface and performs deformation using molecular modelling method. Deformation characteristics of the kidney is represented by the free parameters of the molecular modelling method. The deformation algorithm produces realistic results in real time.
\end{abstract}

Keywords: deformation simulation, kidney model, molecular modelling method.

\section{Introduction}

Applications of computer graphics especially in the area of surgical simulations become very popular in recent years. This specific application requires various techniques and research topics including, heptic interaction, collision detection, 3D visualization and soft tissue deformation. Among these research areas, soft tissue deformation and simulation plays the central role in such applications.

Researchers have been developing number of methods to simulate deformation as realistic as possible and in real time $^{(1)}$. Among them, two methods become very popular: Finite element modelling (FEM) and Mass Spring Systems (MSS) $)^{(1,2)}$. Most accurate results are achieved by using FEM. FEM therefore is used in many applications ${ }^{(3,4,5)}$. Researchers have developed techniques to improve accuracy $^{(6)}$ and speed ${ }^{(7)}$ of FEM while there are number of works reported on improving accuracy ${ }^{(8)}$ and sped $^{(9)}$ of MSS.

Therefore, whilst much research effort has been spent on improving such techniques in the area of physical accuracy and performance, other methods, such as the ChainMail algorithm ${ }^{(10)}$, Mass Spring Chain ${ }^{(11)}$ and Molecular Modelling Method ${ }^{(12)}$ have been proposed aiming at interactive frame rates. This paper will explore the use of molecular modeling method and apply the method for deformation simulation of kidney for stone removal.

\section{Molecular Modelling Method}

Mass spring system uses internal forces affecting on each mass points and then uses Euler integration method to calculate acceleration, velocity and displacements. Similarly, particle systems work based on potential energies affecting each particle. Based on total potential energy, forces affecting each particle are determined and then displacements are again calculated using Euler integration.

One of the first application of molecular modelling method for deformation simulation employed Lennard-Jones potential formulation to calculate forces between atoms ${ }^{(13)}$. This technique is explored in more detail and applied to various deformation simulation using different potential formulations between atoms ${ }^{(14,15)}$.

Molecular modelling describes atoms with its covalent and non-covalent bonds and calculates the potentials based on this structure. In molecular modeling method, only covalent bonds are used to calculate the potentials and resulting forces. In this technique, deformable body is 


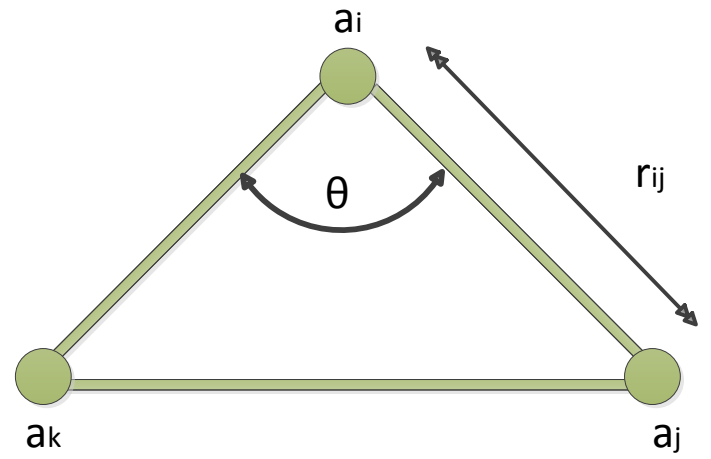

Fig. 1. A triangle element with its bond angle, $\theta$, and bond length. $\mathrm{r}_{\mathrm{ij}}$.

represented by triangles and vertices of each triangle is considered as atoms. The edges of the triangles are thought to be bonds between atoms. This technique then uses bond length and angles of the triangles to form potentials and to calculate corresponding forces. This structure is shown in figure 1 .

There are three potential function used in this representation. The potential energy for bond stretching and compressing is given as,

$$
\phi_{b}=\sum_{1,2 \text { pair }} \frac{1}{2} k_{b}\left(r_{b}-r_{0}\right)^{2}
$$

Where $k_{b}$ is the bond force constant, $r_{b}$ is the bond length and $r^{0}$ is the bond rest length. The resultant force can be obtained using negative gradient of the potential function as,

$$
\begin{array}{r}
f_{b}=-k_{b}\left(r_{i j}-r_{i j}^{0}\right) \overrightarrow{r_{l j}}+ \\
k_{b}\left(r_{i k}-r_{i k}^{0}\right) \overrightarrow{r_{l k}}+ \\
k_{b}\left(\left(r_{j k}-r_{j k}^{0}\right) \overrightarrow{r_{j k}}\right.
\end{array}
$$

Where $\vec{r}$ shows the force direction. The potential energy due to bond angle bending is given by the following function,

$$
\phi_{\theta}=\sum_{\text {bond }} \frac{1}{2} k_{\theta}\left(\cos \theta-\cos \theta^{0}\right)^{2}
$$

Where $k_{\theta}$ is the angular constant, $\sin \theta$ is bond angle and $\sin \theta^{0}$ is the bond initial angle. The force is then given as,

$$
f_{\theta}=-k_{\theta}\left[\cos \theta-\cos \theta^{0}\right] \frac{r_{j k}}{r_{i k} \cdot r_{i j}} \overrightarrow{r_{j k}}
$$

Another potential function is also defined to take bond angle and length dynamics, acting together as stretch-bend interaction, into account,

$$
\phi_{b, \theta}=\sum_{t r i}\left(\cos \theta-\cos \theta^{0}\right)\left[\left(r-r^{0}\right)_{a}+\left(r-r^{0}\right)_{b}\right]
$$

Where $a$ and $b$ represents bonds to a common atom. The force corresponding this potential function is then,

$$
\begin{aligned}
f_{b, \theta}= & \frac{r_{j k}}{r_{j k} \cdot r_{i k}}\left[\left(r_{i j}-r_{i j}^{0}\right)+\left(r_{i k}-r_{i k}^{0}\right)\right] \overrightarrow{r_{j k}}+ \\
& \left(\cos \theta-\cos \theta^{0}\right) \overrightarrow{r_{l j}}+ \\
& \left(\cos \theta-\cos \theta^{0}\right) \overrightarrow{r_{l k}}
\end{aligned}
$$

The total force acting on each atom can then be found by summing forces generated by three potential functions as,

$$
f_{\text {int }}=f_{b}+f_{\theta}+f_{b, \theta}
$$

Compared to force generated by mass spring systems, force given by equation 7 takes more parameter into account and thus produces more accurate results. Deformation characteristic of the deformable object is embedded the above equation by the parameter $k_{b}$ and $k_{\theta}$. Since the total internal force is obtained, equation 7 , Euler integration method is used to calculate the displacement of each atom and therefore determine the deformation.

\section{Kidney Model Generation}

Obtaining a suitable mesh model for the kidney is very important. This model should include enough triangle for visually plausible results and for real time performance. This model should also include enough detail inside the kidney itself. Calyx are small areas inside kidney and they may contain rocks. They are modelled in this work as well.

There are two approaches on obtaining the kidney model. One is purely using segmentation. The kidney model is segmented from its surrounding using segmentation tools. Figure 2 shows resultant kidney model after segmentation process. This model obtained using segmentation process is not very convincing esthetically, as can be seen from kidney model given by figure 2 . 


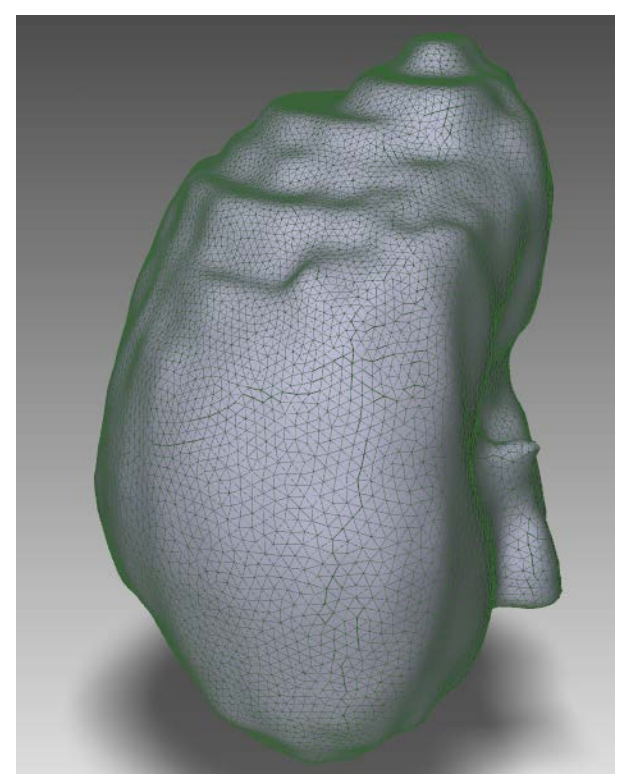

Fig. 2. The kidney model generated by segmentation,

Another approach is using segmentation and modelling programs together to obtain a model. Segmentation is used as guidance and a drawing program is then used to draw a mesh representation of the kidney. The result is completely a geometric model. Figure 3 shows the result of the geometric model obtained in this work. The advantage of this approach is that results is very smooth and visually convincing.

Calyx and a small rock is also modelled as shown in figure 4. Since this model and its deformation

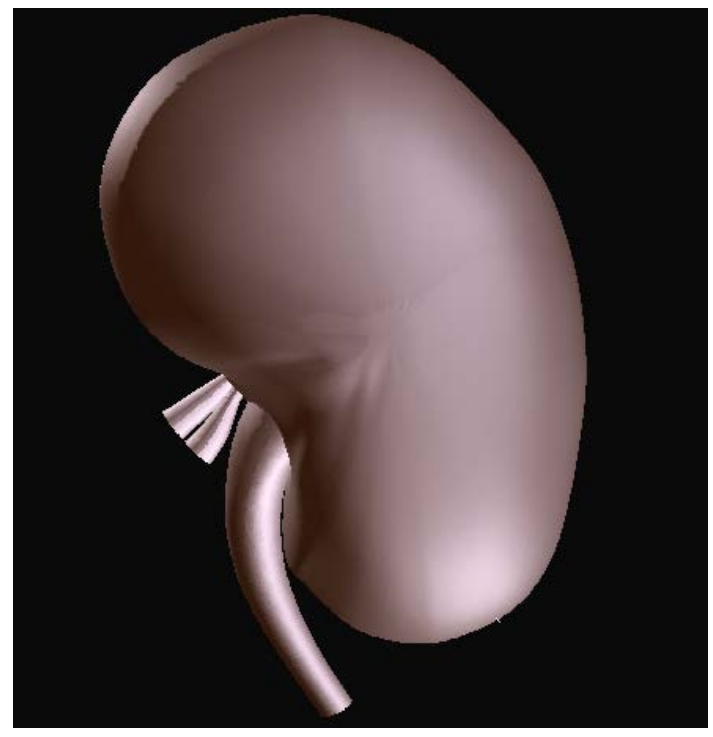

Fig. 3 The geometric kidney model to be used in the simulations.

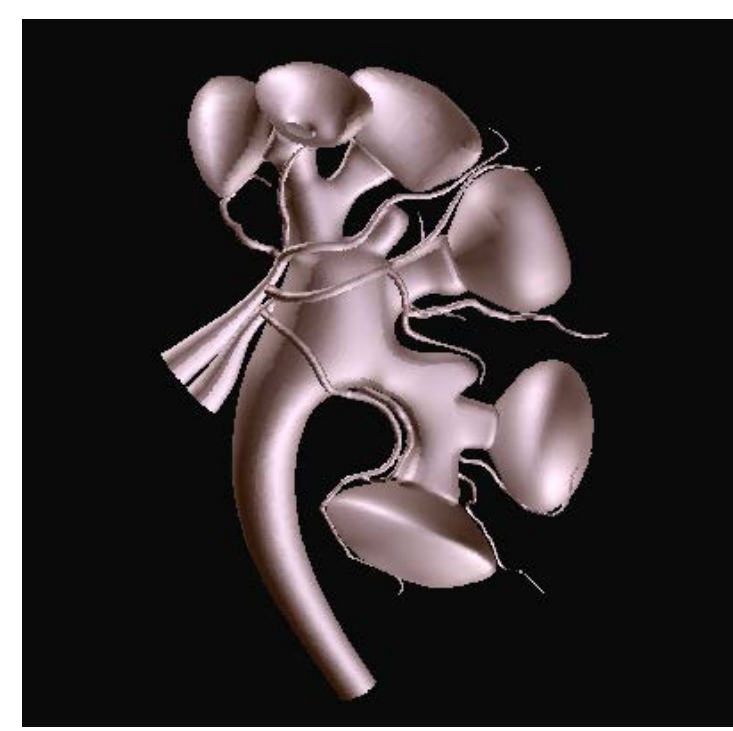

Fig. 4. Calyx are the inside structure of the kidney.

simulation will be used for educational purposes on the simulator, visual convincing is very important.

The kidney model with its surrounding and the rock inside kidney is given in figure 5 , where a small rock can be seen.

\section{Deformation Simulation}

Molecular modelling method is applied to deformation simulation of the kidney. The kidney model used in these simulations consists of 1.986 vertices and 3.968 triangles. A surface model is used.

A surgical tool, Nephroscope, is used, as in real surgery, to perform the kidney rock removal. Figure 6

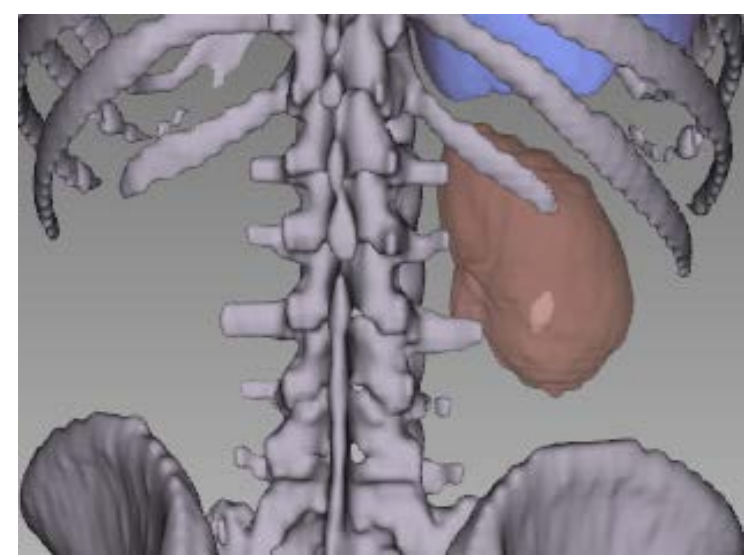

Fig. 5. A small rock inside the kidney. 


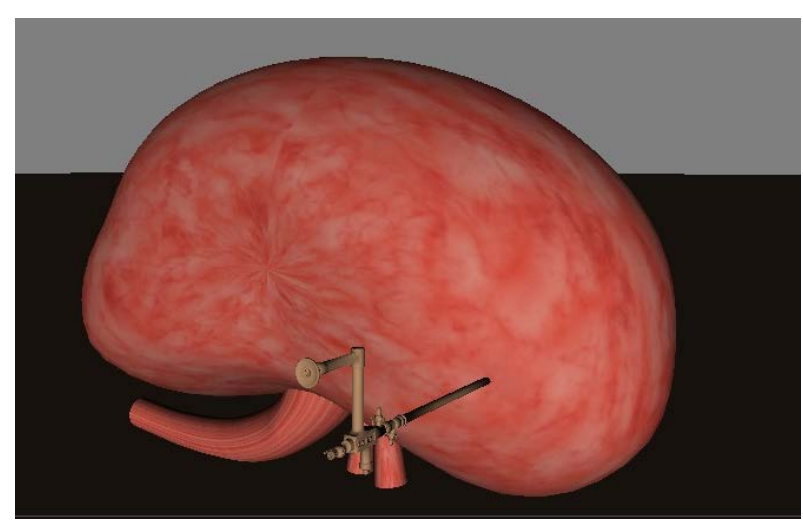

Fig. 6. The kidney and Nephroscope interaction.

shows Nephroscope and kidney before the deformation

Collision between the Nephroscope and the kidney is detected and then molecular modeling method is used to perform deformation. Deformed kidney is shown in figure 7. As can be seen from the figure, molecular modelling method produces visually convincing results. This result is obtained in real time for given mesh representation.

\section{Conclusions}

This paper demonstrates the effective use of molecular modelling technique, applied to kidney deformation. Molecular modelling method takes more parameter, bond length and angle, compared to mass spring systems, which only uses bond length. While this method produces accurate results it also shows real time performance. This method is successfully applied to kidney surgery simulation.

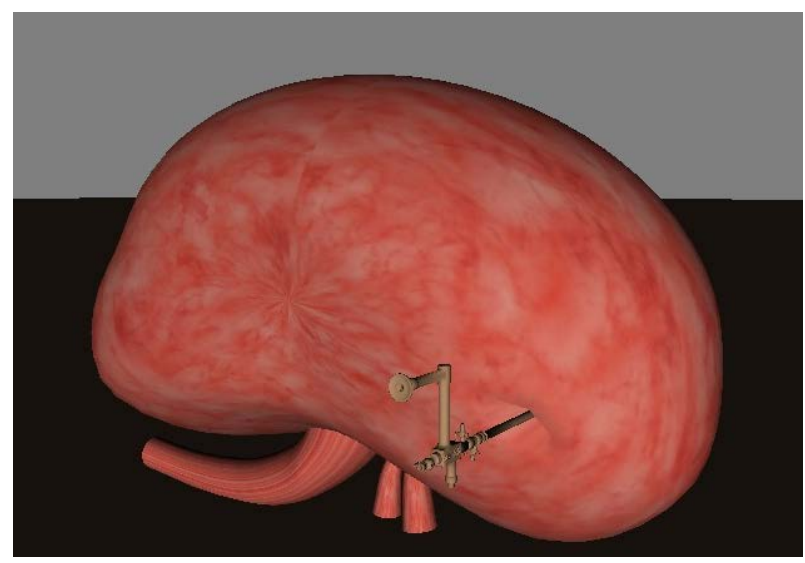

Fig. 7. Collision is detected and deformation is performed.

\section{Acknowledgment}

This work is founded by TUBITAK, The Scientific and Technological Research Council of Turkey, with project number 1130222

\section{References}

(1) Nealen, A., Müller, M., Keiser, R., Boxermann, E., Carlson, M.: "Physically Based Deformable Models in Computer Graphics”, Proceedings of Eurographics, pp.71-94, 2005

(2) W. Mollemans, F. Schutyser, N.Nadjimi, F. Maes, P. Suetens: "Predicting soft tissue deformations for a maxillofacial surgery planning system: From computational strategies to a complete clinical validation”, Medical Image Analysis, Vol. 11, Issue 3, pp. 282-301, 2007

(3) M. Bro-Neilsen: "Finite Element Modeling in Surgery Simulation”, Proceedings of the IEEE, Vol. 86, No. 3, pp. 490-503, 1998

(4) R. M. Koch, S.H.M. Roth, M.H. Grass, A. P. Zimmermann, H. F. Soiler: “A framework for facial surgery simulation”, ETH Zurich, CS Technical Report \#326, Institute of Scientific Computing, June 18, 1999

(5) Teeranoot Chanthasopeephan, Jaydev P. Desai , Alan C., W. Lau: "Modeling soft-tissue deformation prior to cutting for surgical simulation finite element analysis and study of cutting parameters" IEEE Transactions on Biomedical Engineering, Vol. 54 , Issue: 3 pp. 349 - 359, 2007

(6) Wu, X., Downes, M.S., Goktekin, T., Tendick, F.: “Adaptive nonlinear finite elementsn for deformable body”, 30 Int'l Conf. Computer Graphics and Virtual Reality | CGVR'09 simulation using dynamic progressive meshes,.Eurographics, Vol. 20, pp. 349-358, 2001

(7) G. Debunne, M. Desbrun, M.P. Cani, A. Barr: "Dynamic realtime deformations using space and time adaptive sampling”, Computer Graphics proceedings, proceeding of Siggraph, Aug 2001

(8) B.A. Lloyd, G. Szekely, M. Harders: "Identification of spring parameters for deformable object simulation” IEEE Transaction on Visualization on Computer Graphics, Vol. 13, No. 5, pp. 10811094, 2007

(9) Kang, Y. M., Choi, J. H., Cho, H. G.: "Fast and stable animation of cloth with an approximated implicit method" Proceedings of the Computer Graphics International(CGI’00), pp. 247-255, 2000

(10) S. GIBSON: "Using Linked Volumes to Model Object Collisions, Deformation, Cutting, Carving, and Joining”. IEEE Transactions on Visualization and Computer Graphics, Vol. 5, No 4, 1999 
(11) Duysak, A., Zhang, J. J.: "Fast Simulation of Deformable Objects. International symposium on Computer Animation”, The 8th International Conference on Information Visualization, IEEE Computer Society, pp. 422-427, 2004

(12) A. Sait Sekercioglu, Alpaslan Duysak: "Molecular Modeling for Deformable Object Simulation”, CGVR, The International Conference on Computer Graphics and Virtual Reality, pp. 28-31, July 13-16, USA, 2009

(13) M. Pithioux, O. Lopez, U. Meier, C. Monserrat, M.C. Juan, M.Lcaniz, ParSys: "a new particle system for the introduction of on-line physical behaviour to threedimensional synthetic objects” Computer\&Graphics, pp. 135-144, 2005

(14) A. Sait Sekercioglu and Alpaslan Duysak: “Application of molecular modeling with mass-spring systems for computer simulation and animation” International Journal of Physical Sciences Vol. 4 (9), pp. 500-504, 2009

(15) A. Sait Sekercioglu, Alpaslan Duysak: “Molecular Modeling for Deformable Object Simulation. CGVR, The International Conference on Computer Graphics and Virtual Reality, pp. 28-31, July 13-16, USA, 2009 